\title{
CHEMICAL AND MECHANICAL DAMAGE IN CONCRETE DUE TO SWELLING OF ALKALI-SILICA GEL
}

\author{
C. Comi ${ }^{1}$, P. J. M. Monteiro ${ }^{2}$, R. Pignatelli ${ }^{1}$ \\ ${ }^{1}$ Department of Structural Engineering, Politecnico di Milano (claudia.comi@ polimi.it) \\ ${ }^{2}$ Department of Civil and Environmental Engineering, University of California Berkeley
}

\begin{abstract}
In the present work, concrete affected by alkali-silica reaction (ASR) is represented as a two-phase material made of a solid skeleton and an expanding gel, which exerts a pressure capable of severely damaging concrete. As suggested by the experimental results, degradation due to ASR is considered to be localized around the reactive sites and it is described through an isotropic chemical damage variable. Another internal variable is introduced to describe the mechanical damage induced by the external loads. The chemical damage evolution depends on the reaction extent and it is calibrated using the value of the gel pressure, estimated by applying the electrical double-layer theory to the experimental values of the surface charge density obtained from ASR gel specimens. The model is then employed to simulate compression and flexure tests results reported in the literature.
\end{abstract}

Keywords: ASR, concrete, damage, chemical damage.

\section{INTRODUCTION}

The alkali-silica reaction (ASR) occurs in concrete between some forms of amorphous silica contained in the aggregates and the alkali in the cement paste. The reaction produces an amorphous gel that in the presence of water expands and, after filling the concrete initial porosity, exerts a pressure that can cause severe damage. In [1] the electrical double-layer theory was used to interpret the swelling mechanism of the alkali-silica gel. Here, starting from the experimental data on the surface charge density measured on gel specimens collected in Furnas dam (Brazil), the electrical double-layer theory is applied to estimate the gel pressure exerted on the concrete matrix. The obtained values are in agreement with the values measured with mechanical tests (see e.g. [2] and [3]).

In the literature ([4], [5], [6]) poro-mechanical models have been proposed and successfully used in order to describe the structural effects due to ASR. However with these existing models good results in terms of expansion can only be obtained with values of the gel pressure one order of magnitude higher then the experimental ones. This unrealistic requirement is because all the phases are homogenized and, hence, the deterioration of the concrete skeleton, is smeared homogeneously. A different behavior is observed in concrete structures affected by the reaction: there is a severe damage in the neighborhood of the reactive sites while the overall mechanical properties reduction is limited. Starting from these experimental evidences, the main objective of the present work is the combination of a phenomenological 
approach of poro-mechanics with the experimental information at the micro-scale, elaborated through the double-layer theory.

A two-phase poro-mechanical model is presented, in which the wet gel and the concrete skeleton represent the homogenized layers. In the model the material presents two porosities: the common concrete porosity filled by the gel and an additional porosity due to the ASR chemical damage. An isotropic internal damage variable describes the chemical degradation mechanism, while another independent damage internal variable describes the degradation induced by the external loads.

In order to link the overall coefficient parameters with the microscopic quantities, a simplified microscopic interpretation is proposed. The microscopic chemical damage due to ASR depends on the reaction extent and it is restricted to a small portion of concrete surrounding the pores filled by the gel in pressure while the remaining part of the concrete skeleton is undamaged. The maximum value of the chemical damage and the volume fraction of the damaged portion of concrete are obtained by an identification procedure using the experimental values of the Young's modulus reduction of reacted samples and the values of the gel pressure computed through the diffuse double-layer theory, both referred to free-expansion in saturated conditions. The mechanical damage depends on the overall stress, affects both the concrete skeleton and the gel and allows to model the homogeneously distributed degradation due to external loads. The model is validated by simulating the experimental tests on reacted concrete cylindrical specimens axially loaded and confined with steel rings reported in [7] and the compression tests and the three point bending tests reported in [8].

\section{COMPUTATION OF THE ASR GEL PRESSURE}

Electrical double-layer theory has been used successfully to explain the expansion caused by ASR (see [1]). According to this theory (e.g. [9]) the negative charge of silica particles attracts the alkaline cations dissolved in the pore solution and these ions (sodium or potassium) form a layer around the silica particles. The gel pressure can be interpreted as an osmotic pressure due to the different concentration of cations between the silica surface and the bulk solution.

The theory permits to write the gel pressure $p$ as a function of the surface potential $\mathcal{V}_{m}$ at the midpoint between two charged particles

$$
p=C_{0} R T\left[\exp \left(Z \frac{\mathcal{V}_{m}}{\mathcal{V}_{D}}\right)+\exp \left(-Z \frac{\mathcal{V}_{m}}{\mathcal{V}_{D}}\right)-2\right]
$$

where $Z$ is the valence of the ions, $\mathcal{V}_{D}=R T / F$ is the potential scaling factor, $C_{0}$ is the bulk electrolyte concentration, $F$ is the Faraday constant, $R$ is the molar gas constant and $T$ is the absolute temperature. The value of the surface potential $\mathcal{V}_{m}$, can be expressed in terms of the surface charge density $q$ by solving the following transcendental equation

$$
\tan \left(\frac{\sqrt{\exp \left(\frac{Z \mathcal{V}_{m}}{\mathcal{V}_{D}}\right)}}{2}\right)=-\frac{\chi q(x)}{2 H F} \frac{1}{\sqrt{\exp \left(\frac{Z \mathcal{V}_{m}}{\mathcal{V}_{D}}\right)}}
$$




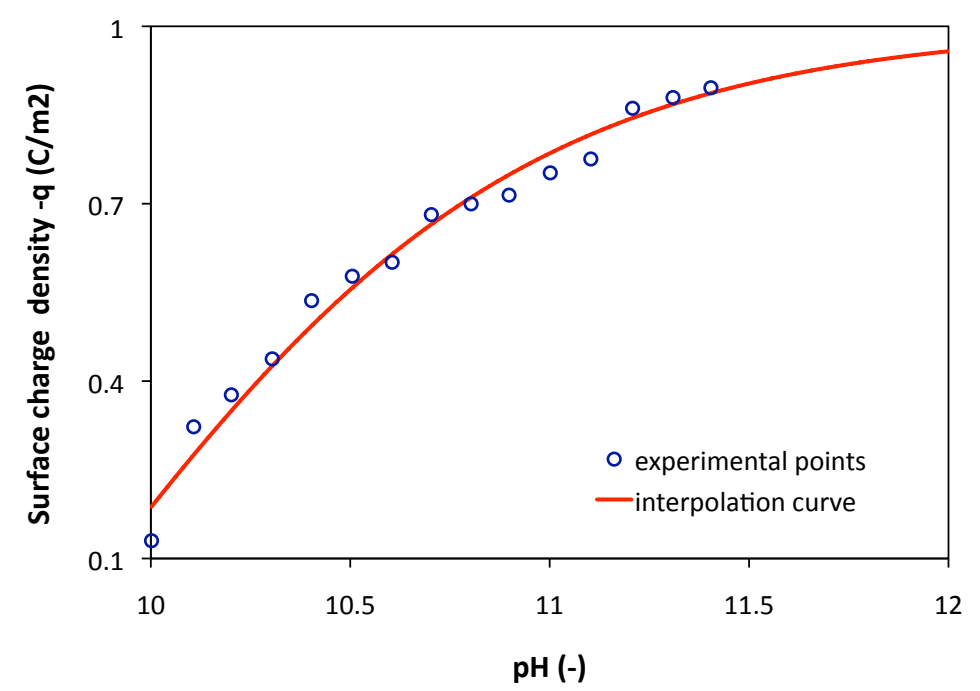

Figure 1. Measured surface charge for the electrolyte solutions of $0.7 \mathrm{~mol} / \mathrm{L} \mathrm{NaCl}$ : experimental data from Furnas dam (Brazil) ASR gel specimens and interpolation curve, extrapolated until $\mathrm{pH}=12$.

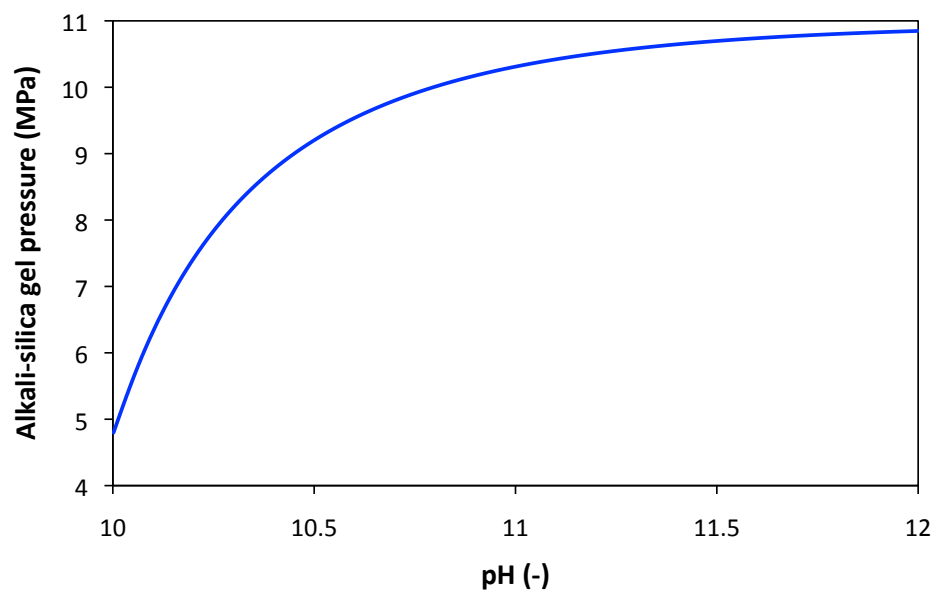

Figure 2. Values of the alkali-silica gel pressure computed by using the double-layer theory and the surface charge density measured on Furnas dam (Brazil) gel samples.

where $\chi=2 F^{2} Z^{2} / \varepsilon_{0} D_{e} R T, \varepsilon_{0}$ and $D_{e}$ are the permittivity of vacuum and the dielectric constant respectively, and $H=\sqrt{\chi C_{0}}$ is the inverse of the "diffuse double-layer theory thickness".

The combination of (1) and (2) permits to compute the gel pressure as a function of the surface charge density, which is a measurable quantity.

We have measured the surface charge density of finely ground particles of alkali-silica gel extracted by the Furnas dam, located on the Rio Grande river in Minas Gerais (Brazil), as a function of $\mathrm{pH}$. The experimental determination of the surface charge density has been carried out through potentiometric tritation in $0.7 \mathrm{~mol} / \mathrm{L}$ electrolyte solution $\mathrm{NaCl}$ until $\mathrm{pH}$ 10 to avoid silica dissolution. Figure 1 shows the experimental results and the interpolating curve until $\mathrm{pH} 12$, representing a more realistic value for the $\mathrm{pH}$ of the concrete pore solution. 


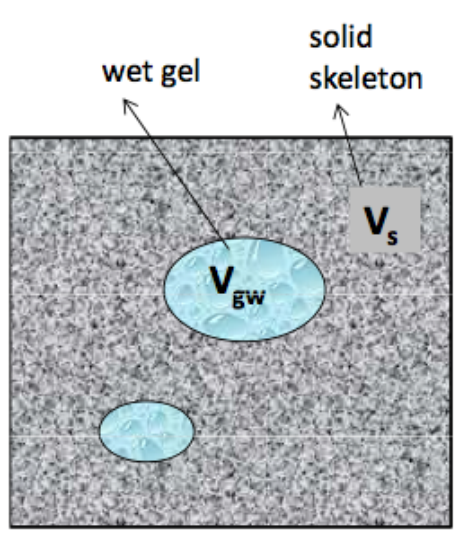

(a)

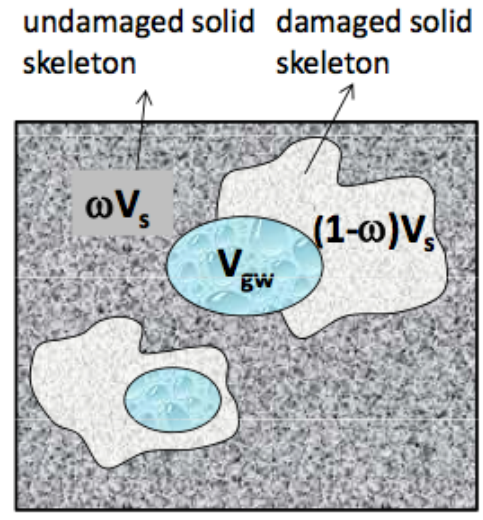

(b)

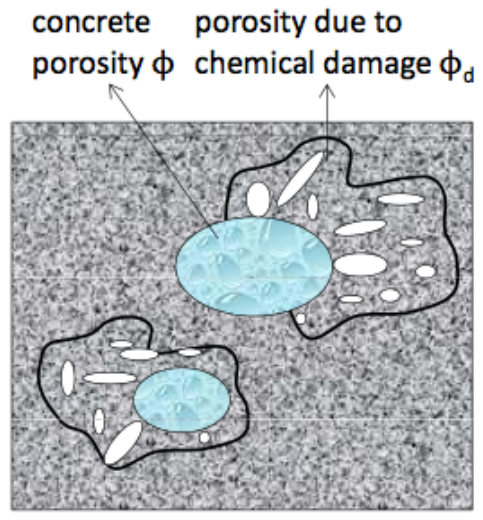

(c)

Figure 3. Schematic representation of the representative volume element.

Figure 2 shows the values of the gel pressure obtained with the relation (1). It must be noted that the values of the gel pressure are obtained with a highly reactive alkaline solution, hence they should be considered as the upper limit for the pressure values.

\section{CHEMICAL AND MECHANICAL DAMAGE MODEL}

In the framework of the mechanics of porous materials ([10], [11]), concrete affected by ASR is interpreted as a two-phase heterogeneous material constituted by the homogenized concrete skeleton $(s)$, that includes the cement paste, the aggregates and the non-connected porosity, and the homogenized wet gel $(g w)$, which consists of the gel produced by the chemical reaction, the adsorbed water and the gas phases (aqueous vapor and dry air) see Figure 3a. The total volume of the representative volume element $V$ can be expressed as the sum of the volumes occupied by the solid and the wet gel phase, namely $V_{s}=V-\phi V_{0}$ and $V_{g w}=\phi V_{0}$, $\phi$ being the total concrete Lagrangian porosity. This porosity, independent from the ASR, will be called primary porosity. With these definitions, the volumetric fraction of the wet gel can be expressed as $\zeta_{g w}=V_{g w} / V$.

The solid skeleton volume $V_{s}$ is divided into a part $\omega V_{s}$ which is not affected by chemical damage and a part $(1-\omega) V_{s}$, representing the reactive sites, which can experience degradation due to ASR, as shown in Figure 3b. The chemical damage $d$ induced by ASR, localized around the reactive sites, is described through an additional porosity $\phi_{d}$ which depends exclusively on the reaction extent and grows only in the damageable portion of the reference volume element, see Figure 3c. According to [12], we assume the following relation between damage and porosity

$$
d=1-\left(1-\phi_{d}\right)^{3}
$$

The homogenized constitutive equations, relating the total stress $\sigma$ and the total strain $\varepsilon$, can be found by combining the strain and stress partition equations

$$
\begin{gathered}
\operatorname{tr} \boldsymbol{\varepsilon}=\left(1-\phi_{0}\right) \omega t r \varepsilon_{s}+\left(1-\phi_{0}\right)(1-\omega) \operatorname{tr} \varepsilon_{d}+\left(\phi-\phi_{0}\right) \\
\boldsymbol{\sigma}=\left(1-\phi_{0}\right) \omega \boldsymbol{\sigma}_{s}+\left(1-\phi_{0}\right)(1-\omega) \boldsymbol{\sigma}_{d}-\phi_{0} p \mathbf{1}
\end{gathered}
$$


the relation between the volumetric strain of the damaged portion $\left(\operatorname{tr} \varepsilon_{d}\right)$ and the volumetric strain of the undamaged portion $\left(\operatorname{tr} \varepsilon_{s}\right)$ of the concrete skeleton

$$
\operatorname{tr} \varepsilon_{s d}=\left(1-\phi_{d 0}\right) \operatorname{tr} \varepsilon_{s}+\phi_{d}-\phi_{d 0}
$$

and the relations between the volumetric stress and the volumetric strain for the undamaged $(s)$ and damaged $(d)$ concrete skeleton portions

$$
\frac{\operatorname{tr} \boldsymbol{\sigma}_{s}}{3}=K_{s} \operatorname{tr} \varepsilon_{s}, \quad \frac{\operatorname{tr} \boldsymbol{\sigma}_{d}}{3}=(1-d) K_{s} \operatorname{tr} \varepsilon_{d}
$$

In equations (4)-(7) $\operatorname{tr} \varepsilon$ is the total volumetric strain and $\sigma$ is the total stress; $p$ the pressure associated with the change in concrete porosity $\left(\phi-\phi_{0}\right)$ filled by the wet gel, $\phi_{0}$ is the initial concrete porosity; $\phi_{d}-\phi_{d 0}$ is the change in the porosity due to chemical damage, $\phi_{d 0}$ the initial value, assumed equal to zero; $K_{s}$ and $(1-d) K_{s}$ are the bulk moduli of the undamaged and damaged portion of the concrete skeleton respectively.

By combining the equations (4)-(7) the mean total stress can be expressed as

$$
\frac{\operatorname{tr} \boldsymbol{\sigma}}{3}=\tilde{K} \operatorname{tr} \varepsilon-\tilde{K}\left(\tilde{\phi}-\phi_{0}\right)-\phi_{0} p
$$

where

$$
\tilde{K}=\left[\omega+(1-\omega)\left(1-\phi_{d}\right)^{3}\right] K_{s}
$$

represents a homogenized bulk modulus of the solid skeleton that takes into account the additional porosity created by ASR and

$$
\tilde{\phi}=\phi+(1-\omega)\left(1-\phi_{0}\right)\left[1-\frac{\left(1-\phi_{d}\right)^{3}}{\omega+(1-\omega)\left(1-\phi_{d}\right)^{3}}\right] \phi_{d}
$$

is the homogenized total porosity, which includes both the primary concrete porosity $\phi$ and the additional porosity $\phi_{d}$ due to the chemical damage.

By assuming that the gel pressure $p$ acts both in the concrete porosity $\phi$ and in the porosity due to micro-cracking $\phi_{d}$ at the same time, the following homogenized constitutive equations can be obtained

$$
\begin{gathered}
\frac{\operatorname{tr} \boldsymbol{\sigma}}{3}=K \operatorname{tr} \varepsilon-b p \\
\tilde{\phi}-\phi_{0}=b \operatorname{tr} \varepsilon+\frac{p}{N}
\end{gathered}
$$

that are formally analogous to the constitutive relations originally proposed in [10] and commonly used in poromechanics.

In (11) and (12) $K$ is the macroscopic bulk modulus, $\tilde{\phi}-\phi_{0}$ is the change in the homogenized total porosity defined in (10), $N$ and $b$ are the Biot's modulus and coefficient.

The definition of $N$ and $b$ springs from the combination of equations (8), (11) and (12), remembering that $\operatorname{tr} \varepsilon$ and $p$ are independent state variables

$$
b=1-\frac{K}{\tilde{K}}, \frac{1}{N}=\frac{b-\phi_{0}}{\tilde{K}}
$$



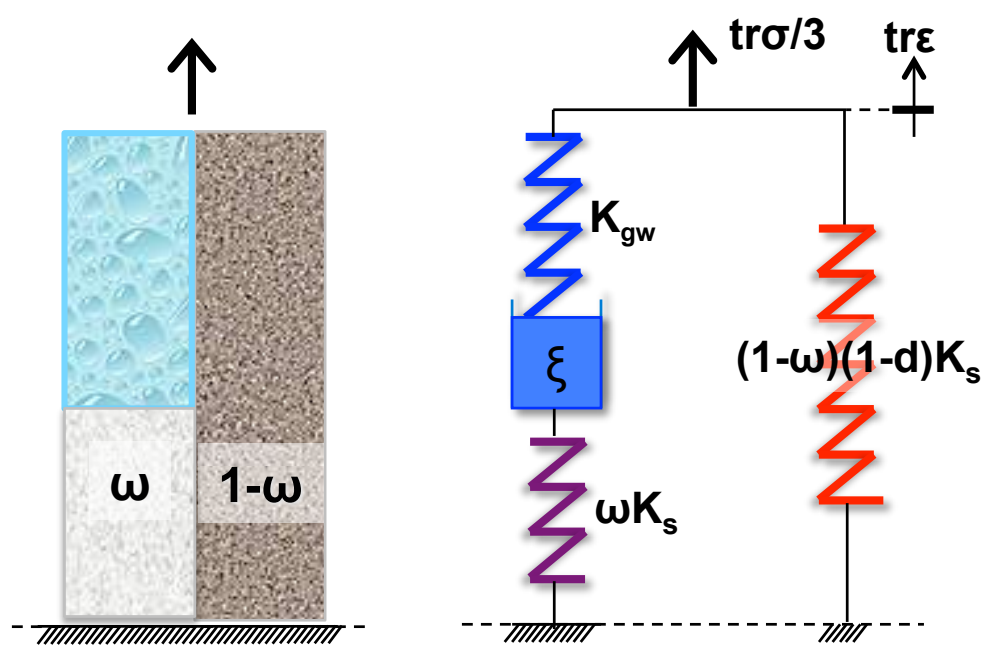

Figure 4. Unidimensional scheme representing the volumetric behavior of the proposed model for concrete damage due to ASR.

The gel pressure $p$ can be related to the change in wet gel volume content $\zeta_{g w}$, instead that to the change in porosity, in this case the constitutive equation (12) can be rewritten as

$$
p=-M\left(b \operatorname{tr} \varepsilon-\zeta_{g w}\right)
$$

where the Biot's modulus $M$ is given by

$$
\frac{1}{M}=\frac{1}{N}+\frac{\phi_{0}}{K_{g w}}
$$

and $K_{g w}$ is the bulk modulus of the fluid (in this case the wet gel) filling the concrete porosity.

Since the experimental measurements of the homogenised bulk modulus $K$ and the Biot's coefficient $b$, necessary to solve the system of equations (11) and (14), are usually not available, the relationship between the macroscopic and microscopic variables of the model can be expressed using a simplified micromechanical scheme.

Figure 4 shows a 1D interpretation of the microscopic behaviour assumed in this work: during its swelling, the gel exerts a pressure on a portion $(1-\omega)$ of the concrete skeleton, working in parallel with the gel, while the rest of the concrete skeleton follows the gel expansion, working in series, without being damaged.

Starting from the equilibrium equations for the system in Figure 4, the following relations can be obtained

$$
\begin{gathered}
\frac{\operatorname{tr} \boldsymbol{\sigma}}{3}=\left[(1-\omega)(1-d) K_{s}\right] \operatorname{tr} \varepsilon-p_{s w} \\
p_{s w}=-\frac{\omega K_{s} K_{g w}}{\omega K_{s}+K_{g w}}(\operatorname{tr} \varepsilon-\bar{c} \xi)
\end{gathered}
$$

where $\bar{c} \xi$ is the expansion of the chemical cell in Figure 4 and $p_{s w}$ is the swelling pressure.

Comparing equations (16) and (17) with equations (11) and (14), identifying the swelling pressure $p_{s w}$ with $b p$ and $\bar{c} \xi$ with $\zeta_{g w}$, one can obtain a relationship between the macroscopic quantities $K$ and $M b^{2}$ and the microscopic bulk moduli $K_{s}$ and $K_{g w}$

$$
K=K(d)=(1-\omega)(1-d) K_{s}
$$




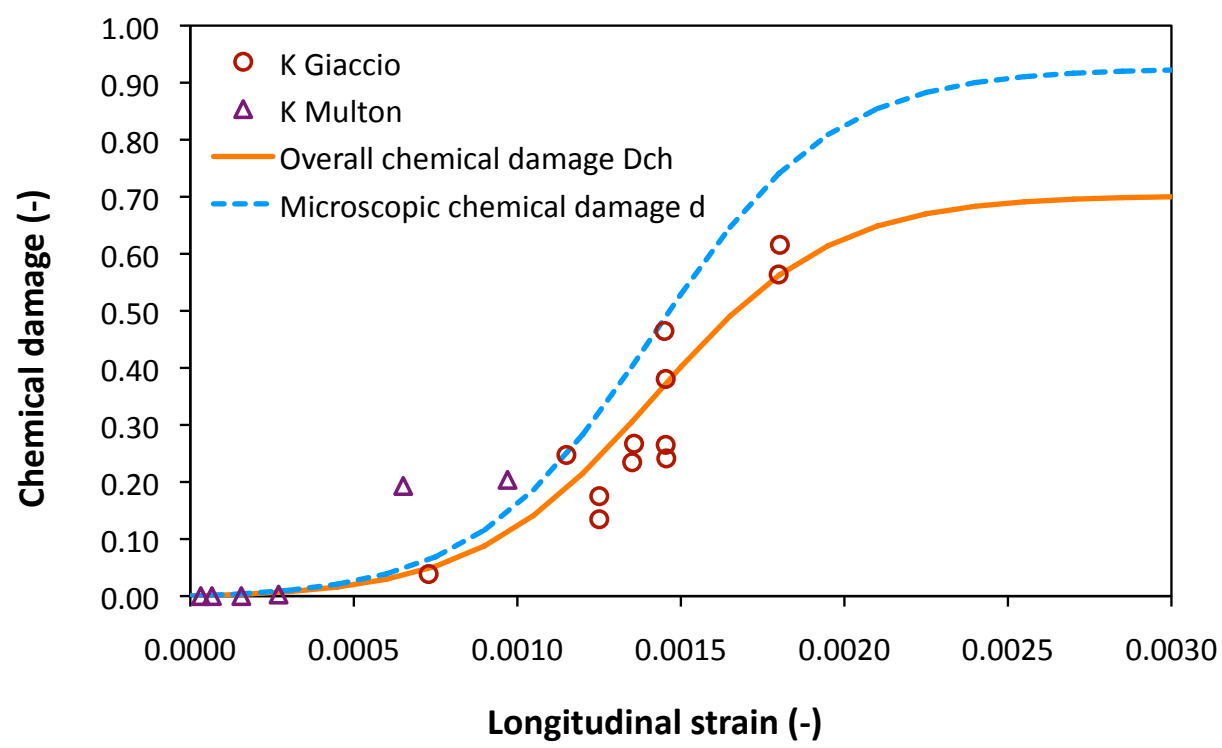

Figure 5. Evolution of microscopic chemical damage $d$ and of macroscopic damage with longitudinal strain in free expansion tests: experimental points from ([8], [7]) and proposed model.

$$
\frac{1}{M b^{2}}=\frac{1}{K_{g w}}+\frac{1}{\omega K_{s}}
$$

By using the equations (18), (9) and (3) into (13), the Biot's coefficient $b$ can be written as functions of the chemical damage $d$

$$
b=1-\frac{(1-\omega)(1-d)}{\omega+(1-\omega)(1-d)}
$$

The above equations show that the introduction of the additional porosity $\phi_{d}$ brings to a different relationship between these macroscopic coefficients and the microscopic variables with respect to their original expression.

The double porosity two-phase model discussed above defines the volumetric behavior of concrete subject to ASR, considering only the chemical damage. For the deviatoric behavior, we assume for the shear modulus the same dependence on the chemical damage obtained for the bulk modulus in (18).

$$
G=G(d)=(1-\omega)(1-d) G_{s}
$$

To model the concrete skeleton degradation due to external loads, we also introduce the internal isotropic damage variable $D=1-\left(1-D_{t}\right)\left(1-D_{c}\right)$, depending on the two scalar variables $D_{t}$ and $D_{c}$, respectively referred to prevailing tension and compression conditions. The state equation, relating the total Cauchy stress $\sigma$, to the conjugate kinematic variable (i.e. the strain of the skeleton $\varepsilon$ ) reads:

$$
\boldsymbol{\sigma}=(1-D)\left\{2 G(d) \mathbf{e}+\left[K(d)(\operatorname{tr} \varepsilon-\alpha \theta)+M b\left(b \operatorname{tr} \varepsilon-\zeta_{g w}-\alpha_{g w} \theta\right)\right] \mathbf{1}\right\}
$$

that can be rewritten as

$$
\boldsymbol{\sigma}=(1-D)[2 G(d) \mathbf{e}+K(d)(\operatorname{tr} \boldsymbol{\varepsilon}-\alpha \theta) \mathbf{1}]-b p \mathbf{1}
$$



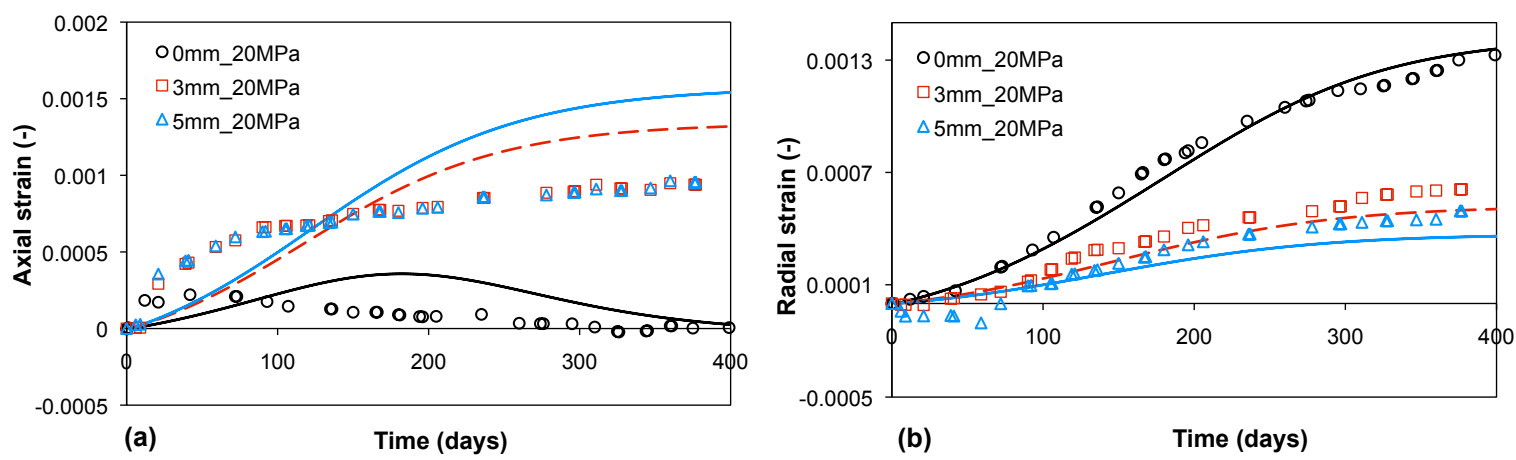

Figure 6. (a) Axial and (b) radial strains of constrained concrete specimens $(3 \mathrm{~mm}$ and $5 \mathrm{~mm}$ steel rings) under compressive $20 \mathrm{MPa}$ load: experimental points from ([7]) and proposed model.

being $p$ the gel pressure

$$
p=-(1-D) M\left[b t r \varepsilon-\zeta_{g w}-\alpha_{g w} \theta\right]
$$

In the above equation $\mathbf{e}$ is the deviatoric strain tensor, $\alpha$ and $\alpha_{g w}$, are respectively the volumetric coefficients of thermal expansion for the concrete skeleton and the wet gel, $\theta$ is the variation of the temperature.

The constitutive model is completed by the evolution equations for the variation of the wet gel volume content $\zeta_{g w}$, the chemical damage $d$ and the mechanical damage $D$. The rate of the gel content is assumed to be proportional to the rate of the reaction extent $\xi$, a phenomenological internal variable $(0 \leq \xi \leq 1)$ used to describe both the gel formation and the gel swelling, considered as simultaneous, in accordance with the experimental results (see e.g. [13]):

$$
\dot{\zeta}_{g w}=c \dot{\xi}
$$

The constant $c$ in (25) is proportional to the free asymptotic volumetric expansion in the isothermal fully saturated case $\epsilon_{A S R}^{\infty}$. Its expression can be obtained from (11) and (14) by imposing the free-stress expansion condition $(\sigma=0)$ and reads

$$
c=\frac{K+M b^{2}}{M b} \epsilon_{A S R}^{\infty}
$$

The reaction extent evolution depends on the histories of temperature and degree of saturation; the form proposed in [6] can be assumed.

The chemical damage $d$ can be expressed as a function of the reaction extent $\xi$ :

$$
d=\frac{1-\exp \left(-r_{1} \xi\right)}{1+\exp \left(-r_{1} \xi+r_{2}\right)} r_{3}
$$

The material parameters $r_{1}, r_{2}$ and $r_{3}$ in (27) and the amount of the damaged concrete skeleton $(1-\omega)$ can be found by equalizing the gel pressure defined in (24) with the value computed through the double-layer theory and shown in Figure 2 and by relating the microscopic chemical damage $d$ to the overall chemical damage $D_{c h}=1-\left(K(d)+M b^{2}\right) /\left(K_{0}+M b^{2}\right)$ :

$$
d=1-\frac{\left(1-D_{c h}\right)\left[(1-\omega) K_{s}+M b^{2}\right]-M b^{2}}{(1-\omega) K_{s}}
$$




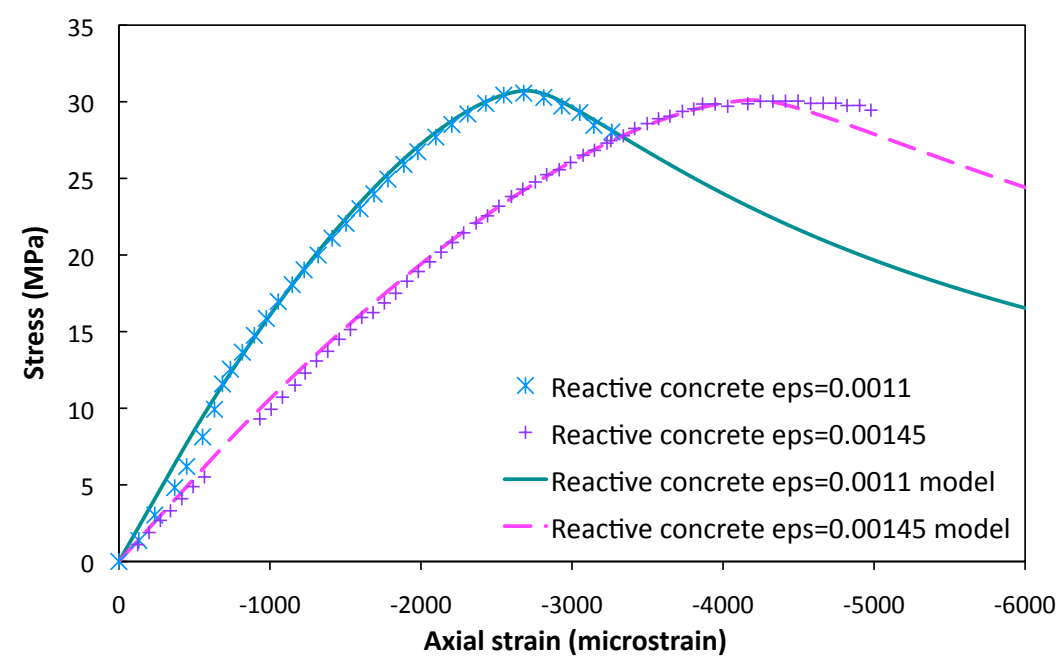

Figure 7. Stress-strain relation in compression for a reactive mixture with time: experimental curves by [8] and proposed model.

The experimental values of the overall chemical damage obtained in free expansion condition and the microscopic chemical damage in (27) are shown in Figure 5.

The evolution of the mechanical damage variables in tension $D_{t}$ and in compression $D_{c}$ is governed by loading-unloading conditions defined in terms of the macroscopic stress $\boldsymbol{\sigma}$. The loading-unloading conditions read

$$
f_{i} \leq 0 \quad \dot{D}_{i} \geq 0 \quad f_{i} \dot{D}_{i}=0, i=t, c
$$

where $f_{t}$ and $f_{c}$ are the damage activation functions in tension and compression, defined as

$$
f_{i}=\frac{1}{2} \mathbf{s}: \mathbf{s}+a_{i 0}(\operatorname{tr} \boldsymbol{\sigma})^{2}+a_{i 1} \operatorname{tr} \boldsymbol{\sigma} h_{i}-a_{i 2} h_{i}^{2}, \quad i=t, c
$$

where $\mathbf{s}$ is the deviatoric stress, $h_{t}$ and $h_{c}$ are the hardening-softening functions and $a_{i 0}, a_{i 1}, a_{i 2}$ $(i=t, c)$ are material parameters governing the shape and dimensions of the elastic domain (see [14] for details).

\section{MODEL VALIDATION}

The present model for the chemical damage can be validated by simulating the tests on loaded (20 MPa) and confined (3 $\mathrm{mm}$ and $5 \mathrm{~mm}$ steel rings) reactive cylindrical specimens reported in [7]. Figures 6 compares the axial and radial experimental results with the ones predicted by the present model. The agreement is quite good, especially if one considers that the model has not been calibrated with the strains obtained in tests on loaded specimens, but only with the experimental value of the gel pressure, the overall Young's modulus reduction and the asymptotic expansion in free expansion tests.

In order to validate the coupling between chemical and mechanical damage model, the uniaxial compression tests on cylinders and the three-point bending tests on notched specimens reported in [8] for samples previously kept in air in saturated conditions until they reached linear expansions in the range of $0.11-0.18 \%$ have been simulated. The results in 
(a)

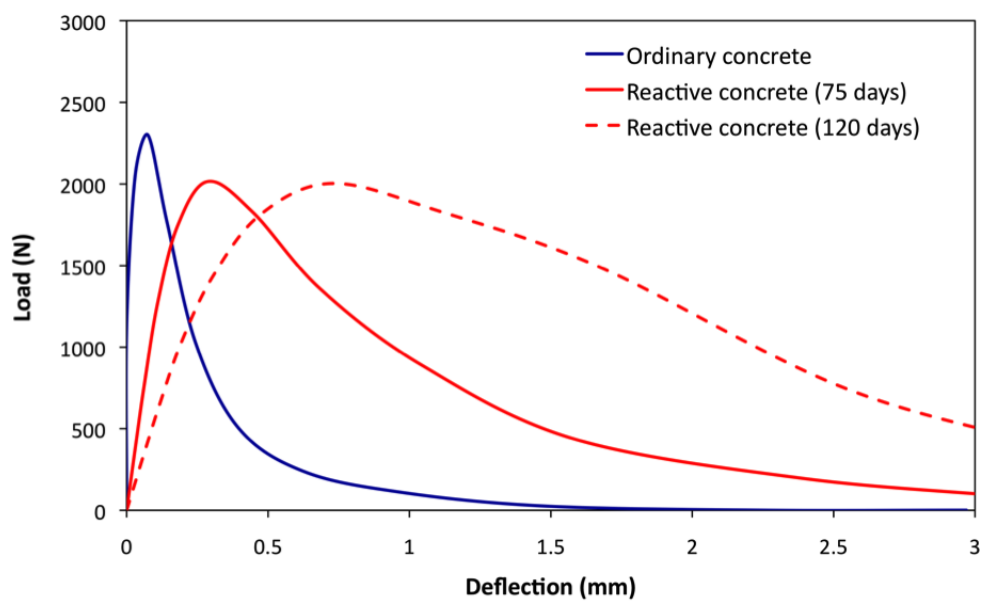

(b)

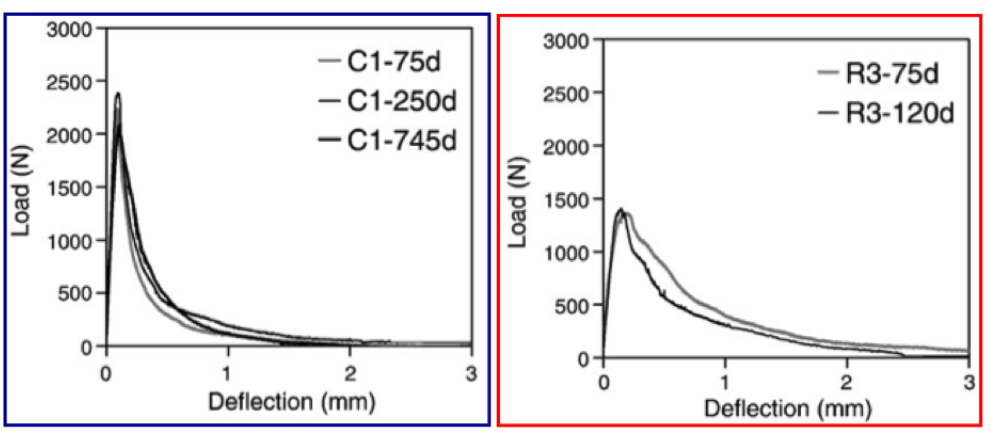

Figure 8. Flexure test on ordinary and reactive concretes: (a) mesh, (b) curves obtained with the model at different time and (c) experimental results by [8]

[8] have been obtained considering various concrete mixtures and show very different overall behaviors according to the constituents of concrete. Since the current model is not able to take into account the chemical composition of the concrete mixtures, the simulations have been performed by considering the mechanical properties of the ordinary reference concrete and the kinetic of the more reactive concrete mixture used in [8] in order to observe the effect caused by the activation of ASR. Figure 7 shows that the model correctly represents the stiffness degradation due to the evolution of the ASR. The reactive concrete used in this example doesn't show a compressive strength reduction. Figure 8a shows the load-deflection curves obtained with the model for the same ordinary and reactive mixtures of the previous example, Figure $8 b$ reports the experimental results for a reference ordinary concrete (C1) and the reactive concrete (R3). The damage induced by ASR in the reactive specimen produces an increased non-linearity before the peak and a more gradual softening in the descending branch. The model is able to reproduce these effcts, but it is not able to correctly represent the reduction in the tensile strength. This effect could be possibly introduced in the model by modifying the activation of the mechanical damage in order to include the influence of the chemical damage. However one should consider that the difference in mixture between rective and non-reactive concrete can also affect the initial tensile strength and the variability in the experimental results reported in [8] makes the modeling of this aspect very difficult. 


\section{CONCLUSIONS}

In the present work we have proposed a new model for the description of degradation in concrete subject to ASR. Two damage mechanisms, the one induced by the chemical reaction and the one induced by the external loads, are considered and described by two independent internal variables. This choice allows for the correct prediction of the overall behaviour and of the swelling pressure. A microscopic scheme has been proposed to interpret the relationship between the behavior of the material at the micro-scale and the overall consequences. In this paper the model has been applied to isothermal problems with a fixed degree of saturation, but the model can also be used in the presence of varying humidity and temperature conditions.

\section{References}

[1] M. Prezzi, P. J. M. Monteiro, and G. Sposito, "The alkali-silica reation, part 1: use of the double-layer theory to explain the behavior of reaction-product gels," ACI materials journal, vol. 94, no. 1, pp. 10-17, 1997.

[2] L. J. Struble and S. Diamonds, "Swelling properties of synthetic alkali silica gels," Journal of American Ceramic Society, vol. 64, no. 11, pp. 652-655, 1981.

[3] M. Kawamura and K. Iwahori, "Asr gel composition and expansive pressure in mortars under restraint," Cement and Concrete Composites, vol. 26, pp. 47-56, 2004.

[4] F. J. Ulm, O. Coussy, L. Kefei, and C. Larive, "Thermo-chemo-mechanics of asr expansion in concrete structures," ASCE Journal of Engineering Mechanics, vol. 126, no. 3, pp. 233-242, 2000.

[5] C. Comi, R. Fedele, and U. Perego, "A chemo-thermo-damage model for the analysis of concrete dams affected by alkali-silica reaction," Mechanics of Materials, vol. 41, pp. 210-230, 2009.

[6] C. Comi and R. Pignatelli, "A three-phase model for damage induced by asr in concrete structures," in IV International Conference on Computational Methods for Coupled Problems in Science and Engineering, 2011.

[7] S. Multon, Evaluation experimentale et theorique des effects mechaniques de l'alcalireaction sur des structures modeles. PhD thesis, LCPC, 2003.

[8] G. Giaccio, R. Zerbino, J. Ponce, and O. Batic, "Mechanical behavior of concretes damaged by alkali-silica reaction," Cement and Concrete Research, vol. 38, pp. 993-1004, 2008 .

[9] J. Israelachvili, Intermolecular and surface forces. Academic press, 1992.

[10] M. A. Biot and D. G. Willis, "The elastic coefficients of the theory of consolidation," Journal of applied mechanics, vol. 57, no. APM44, pp. 594-601, 1957. 
[11] O. Coussy, Poromechanics. New York: John Wiley and Sons, 2004.

[12] K. Kendall, A. J. Howard, and J. D. Birchall, "The relation between porosity, microstructure and strength, and the approach to cement-based materials," Philos. Trans. R. Soc. London, vol. A 310, pp. 139-153, 1983.

[13] M. Ben Haha, G. A. Gallucci, E., and S. K. L., "Relation of expansion due to alkali silica reaction to the degree of reaction measured by sem image analysis," Cement and Concrete Research, vol. 37, pp. 1206-1214, 2007.

[14] C. Comi and U. Perego, "Fracture energy based bi-dissipative damage model for concrete," International Journal of Solids and Structures, vol. 38, pp. 6427-6454, 2001. 